\title{
Precise time and frequency transfer combined BeiDou's RDSS and RNSS signals
}

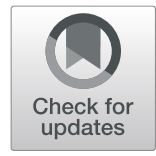

Wenxue Liu ${ }^{1,2}$, Hong Yuan ${ }^{1 *}$, Jian $\mathrm{Ge}^{1}$ and Ying $\mathrm{Xu}^{1}$

\begin{abstract}
Unlike other satellite navigation systems such as GPS (Global Positioning System), the BeiDou satellite navigation system broadcasts RDSS (Radio Determination Satellite Service) and RNSS (Radio Navigation Satellite Service) signals simultaneously on its GEO (geostationary earth orbit) satellites and provides related navigation services. This paper studies the method of using the RDSS and RNSS signals of BeiDou to achieve accurate frequency and time transmission. We analyze the generation mechanism of RDSS signal and RNSS signal of BeiDou GEO satellite, establish a mathematical model of RDSS and RNSS signal frequency transfer, and derive an equation based on BeiDou's RDSS and RNSS signals for accurate frequency and time transmission. We also verified the relevant performance of the method through computer simulation. The results show that the combination of RDSS and RNSS signals from the BeiDou satellite system provides a new solution for its application in precise time and frequency transmission. This method is different from other satellite navigation systems such as GPS and is unique to the BeiDou system, with high accuracy and low dependence on satellite orbit accuracy.
\end{abstract}

Keywords: BeiDou, Precise time and frequency transfer, RNSS, RDSS

\section{Introduction}

China is currently developing its global satellite navigation system, that is, BeiDou Navigation Satellite System, with the abbreviation BDS. By the end of 2012, the regional positioning and navigation service has been provided for users throughout the AsiaPacific area, and the global networking system is currently under construction. Currently, BeiDou comprises five GEO satellites, five IGSO (inclined geosynchronous orbit) satellites, and four medium earth orbit (MEO) satellites [1,2]. Compared with GPS and GLONASS, BDS not only contains MEO satellites, but also includes GEO and IGSO satellites, and simultaneously broadcasts RDSS and multi-frequency RNSS signals on the GEO satellites, and the RDSS and RNSS signals share the same clock source on the satellite. We found that the joint application of the
RDSS and RNSS signals of the BeiDou's GEO satellite can realize a new precise time and frequency transfer method. This method is different from the traditional GPS method.

GPS CV (common view) time and frequency transfer were proposed by Allan and Weiss [3], which uses the C/A pseudorange observable and explicit differencing of the GPS data collected at the two timing laboratories. The time transfer accuracy of this method can be achieved a few nanoseconds. The other ultra-precision time transfer techniques include GPS PPP (Precise Point Positioning time transfer), GPS CP (carrier phase) time transfer, and GPS AV (ALL in View) [4-7]. A study based on the GEO satellite will promote the application study on BeiDou [8]. The paper introduced the GEO navigation satellite to common view time transfer and discussed in

\footnotetext{
* Correspondence: yuanh@aoe.ac.cn

${ }^{1}$ Academy of Opto-Electronics, Chinese Academy of Sciences, No. 9, Deng Zhuang South Road, HaiDian District, Beijing 100094, People's Republic of China

Full list of author information is available at the end of the article
} 
details; the CV method of GEO navigation satellite is also using RNSS signal and similar to the GPS CV method.

In this paper, we propose a method of BeiDou's satellite RDSS and RNSS joint precision time and frequency transmission. This method is different from the traditional GPS CV method and uses BeiDou's RDSS and RNSS signals jointly. It is based on the characteristics of BeiDou RDSS/RNSS signals in satellite common clock source and relevance of signal propagation paths. We also establish the mathematical equations for precise time and frequency transmission between the MCS (Master Control Station) and the satellite; the MCS and the user give the relevant computer simulation results to verify the effectiveness of the method.

\section{RDSS and RNSS signal propagation model}

Distinct from other GNSS (Global Navigation Satellite System), BDS has both RNSS and RDSS signals on the GEO satellite $[9,10]$. Figure 1 shows a schematic diagram of BeiDou's RDSS and RNSS on its GEO satellite.

As illustrated in Fig. 1, BeiDou's GEO satellites provide RDSS information in addition to three RNSS signals. The RNSS signals are generated on satellites similar to GPS signals, but the RDSS signal is different. The signal is generated by the MCS and forwarded to the user via the satellite (path 1 and path 2 of the RDSS signal). After receiving the information, the user forwards the information to the MCS through the satellite (path 3 and path 4 of the RDSS signal).

\subsection{Satellite signal carrier frequency generation model of RDSS and RNSS}

RDSS and multi-frequency RNSS signals are simultaneously broadcast on BeiDou's GEO satellites. RDSS and RNSS carrier signals are generated on satellites, which are common to the clock source on the satellite $[11,12]$, where $f_{\text {RNSS }}^{(i)}$ is the carrier frequency of BeiDou's RNSS signal, $B_{i}$ is a known deterministic value in BD's system configuration, $f_{\mathrm{RDSS}}$ is the carrier frequency of BeiDou's RDSS signal which is also a known deterministic value in system configuration and from satellite to user, and $f_{\mathrm{RDSS}}^{U}$ is the carrier frequency of BeiDou's RDSS signal in system configuration which is also a known deterministic value in system configuration and from MCS to satellite. The following mathematical equations can be easily obtained from Fig. 2:

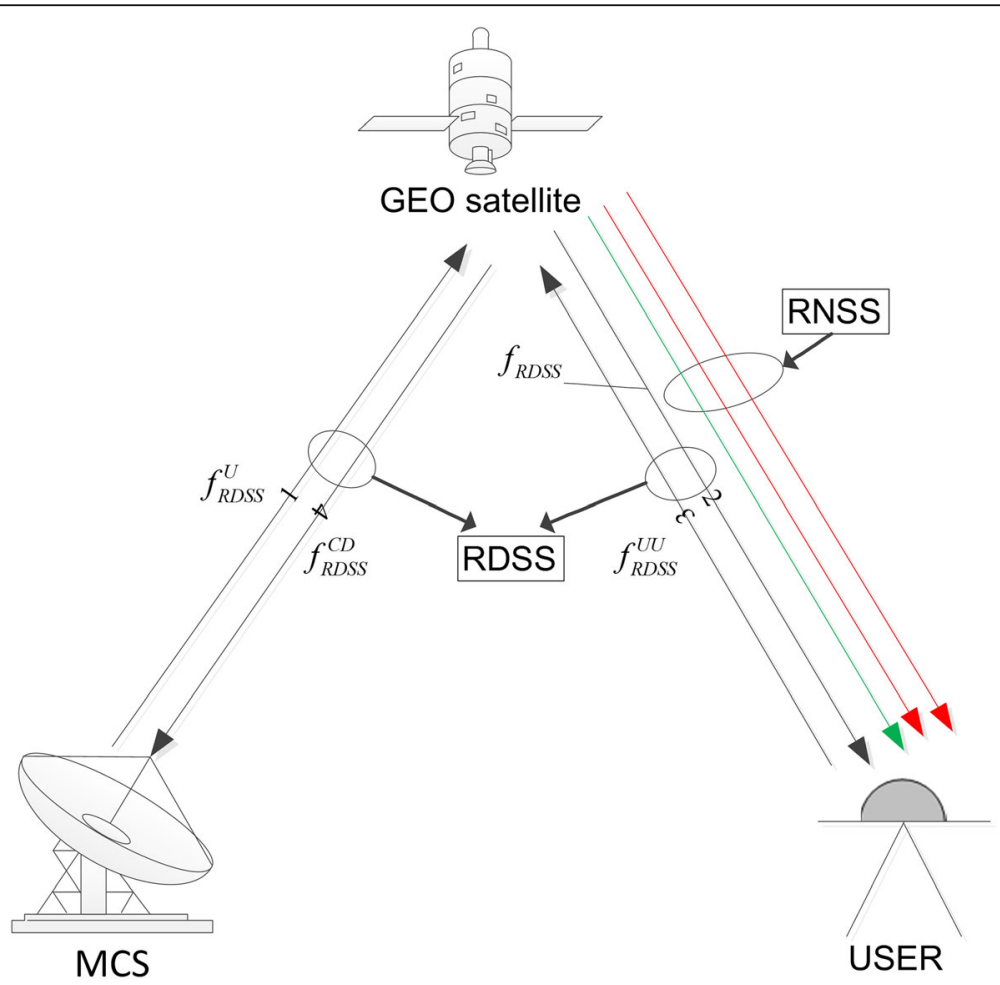

Fig. 1 Schematic diagram of BeiDou's RDSS and RNSS 


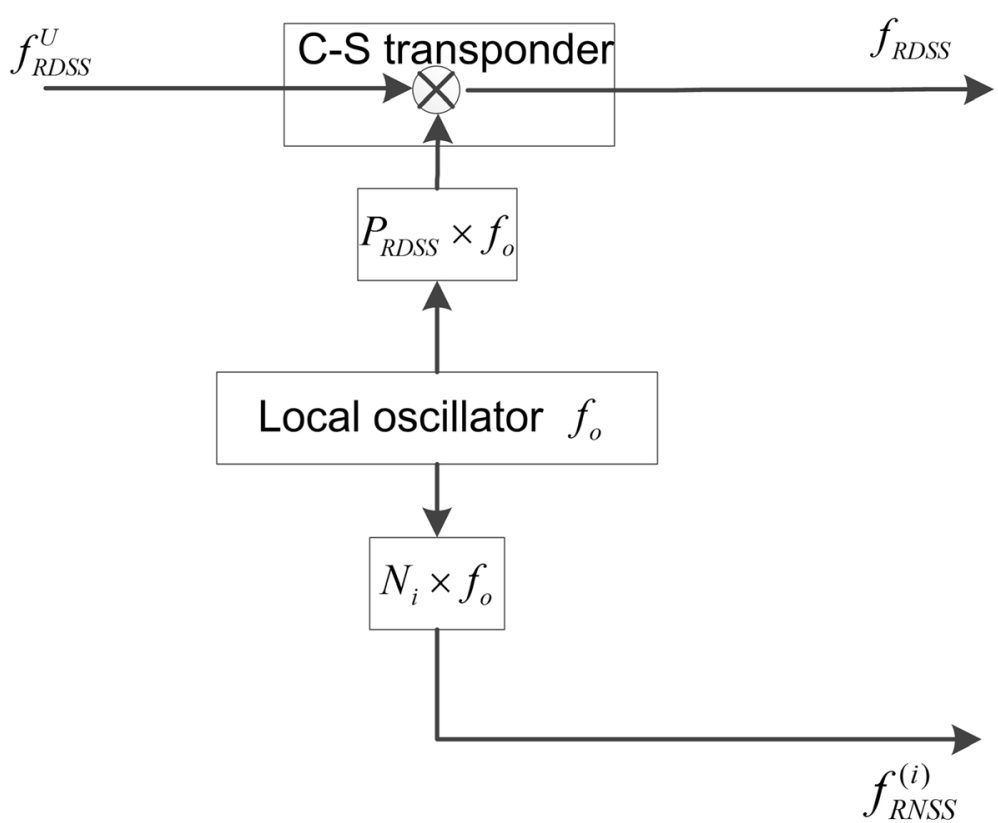

Fig. 2 RDSS and RNSS on-board signal carrier frequency generation

$$
\begin{aligned}
& P_{\mathrm{RDSS}}=\frac{f_{\mathrm{RDSS}}^{U}-f_{\mathrm{RDSS}}}{f_{o}} \\
& N_{i}=\frac{f_{\mathrm{RNSS}}^{(i)}}{f_{o}}
\end{aligned}
$$

where $P_{\mathrm{RDSS}}$ represents the multiple of the satellite C-S responder local oscillator signal and satellite local oscillator signal $f_{o}$, and $N_{i}$ represents the multiple of the satellite RNSS carrier signal and satellite local oscillator signal $f_{o}$. They all are defined as constant in the BeiDou system.

In practice, due to the drift of the atomic clock of the satellite, there is also drift in the frequency of the carrier signal generated by it. Assume that the drift is $\Delta f_{o}$, then the real RDSS and RNSS signal carrier frequencies generated by satellites are changed to the following formula:

$$
\begin{aligned}
& f_{\mathrm{RDSS}}(t)=f_{\mathrm{RDSS}}^{U}-P_{\mathrm{RDSS}} \times\left(f_{o}+\Delta f_{o}(t)\right) \\
& f_{\mathrm{RNSS}}^{(i)}(t)=N_{i} \times\left(f_{o}+\Delta f_{o}(t)\right)
\end{aligned}
$$

Therefore, we obtained the signal frequency generation models of the BeiDou's RDSS and RNSS signals on BeiDou's GEO satellites.

\subsection{Signal frequency models of user received RDSS and RNSS}

After the RDSS and RNSS signals were generated by the BeiDou's GEO satellite, they were transmitted to the receiver, where the RNSS signals were directly generated by the satellites and the RDSS signals were generated by MCS. The section discusses the frequency representation of the BeiDou's RDSS and RNSS signals arriving at the receiver.

\subsubsection{RNSS signal}

The BeiDou's RNSS signal is generated on the satellite, and the satellite signal reaches the user through the ionosphere and troposphere; we assume the $C_{i}$ represents the $1-\mathrm{GHz}$ carrier frequency ionospheric influence factor of RNSS (B1, B2, B3). The modulation on the signal is a time delay in proportion to the number of free electrons encountered and is also (to first order) proportional to the inverse of the carrier frequency squared $\left(1 / f^{2}\right)$ [13]. The phase of the radio frequency carrier is advanced by the same amount. So if we assume the ionospheric influence Doppler on 1 $\mathrm{GHz}$ carrier frequency is $\Delta f_{\mathrm{TEC}}(t)$, then the ionospheric influence factor on the BeiDou signal frequency $f_{\mathrm{RNSS}}^{(i)}$ can be got in the following formula:

$$
C_{i}=\frac{(1 \mathrm{GHz})^{2}}{\left(f_{\mathrm{RNSS}}^{(i)}\right)^{2}}=\frac{10^{18}}{\left(f_{\mathrm{RNSS}}^{(i)}\right)^{2}}
$$

where $i=1,2$, and 3 represents the ionospheric influence factor of the BeiDou's B1, B2, and B3 signal. If we use $\Delta f_{\mathrm{TEC}}$ to represent the influence of the ionosphere on the $1-\mathrm{GHz}$ carrier frequency on the signal path, the frequency of B1 signal affected by the ionosphere can be 
expressed as $C_{1} \times \Delta f_{\text {TEC }}$. Similarly, we can obtain the frequency of other BeiDou RNSS frequency signals affected by the ionosphere.

The troposphere is a region which is not ionized and is not frequency dispersive for frequencies below $15 \mathrm{GHz}$, and the excess group delay is constant with frequency [14]. So the RNSS signal frequency is not changed when the signal gone through the troposphere.

Another change in the carrier frequency of RNSS signals is the relative motion of satellites and user, and its value is positively related to the carrier frequency of the signal. In this paper, if we assume that $k_{i}$ represents the BeiDou's multi-frequency RNSS carrier Doppler frequency coefficient due to the relative motion of the satellite and the user at the $1-\mathrm{GHz}$ signal carrier frequency, then we can get the following equations:

$$
k_{i}=\frac{f_{\mathrm{RNSS}}^{(i)}}{1 \mathrm{GHz}}
$$

where $i=1,2$, and 3 represents the influence factor of the relative motion of satellites and user on BeiDou's B1, B2, and B3 signal. Then, when we assume the influence on the signal frequency at $1 \mathrm{GHz}$ carrier frequency is $\Delta f_{2}(t)$, then the frequency of B1 signal affected by the relative motion can be expressed as $k_{1} \times \Delta f_{2}(t)$. Similarly, we can obtain the Doppler frequency of other BeiDou RNSS frequency signals affected by the relative motion of satellites and user.

The last one is the carrier frequency measurement drift caused by the receiver crystal frequency drift. When the receiver received signal, the receiver measures signal carrier frequency based on the nominal frequency of its own clock which perhaps not its actual crystal frequency. Therefore, the drift of the receiver's own clock will also cause the satellite carrier frequency value measured by the receiver to deviate from the true value, but the influence relationship is relatively simple. We assume that the receiver nominal frequency of its own clock is $f_{o}^{r}$ and its drift is $\Delta f^{\prime}(t)$. Then, in conjunction with the above analysis, we can derive the carrier frequency measured of RNSS signal by the receiver as follows:

$$
\begin{aligned}
f_{\mathrm{RNSS}(i)}^{r}(t)=\left\{N _ { i } \left[f_{o}\right.\right. & \left.+\Delta f_{o}(t)\right]+k_{i} \times \Delta f_{2}(t)+c_{i} \\
& \left.\times \Delta f_{\mathrm{TEC}}(t)\right\} \times \frac{f_{o}^{r}}{f_{o}^{r}+\Delta f_{r}(t)}
\end{aligned}
$$

where $i=1,2$, and 3 represents the signal carrier frequency of BeiDou's B1, B2, and B3.

\subsubsection{RDSS signal}

Different from BeiDou's RNSS signal, BeiDou's RDSS signal was generated by MCS and forwarded to the user via a satellite transponder, and the RNSS signal is generated by the satellite and transmitted to the user. On the BeiDou's GEO satellites, RDSS and RNSS signals use the common atomic clock.

In this paper, we use $f_{o}^{c}$ which represents the MCS atomic clock frequency, which is the standard frequency of BeiDou system, and its frequency is not offset in system. The RDSS uplink signal is from MCS to satellite, which is similar to the RNSS signal, but in the opposite direction. Let $M_{\mathrm{RDSS}}$ represent the ratio of MCS transmit frequency to the MCS atomic clock frequency, $\Delta f_{1}(t)$ represent the uplink carrier Doppler frequency from RDSS signal MCS to satellite, and $\Delta f_{\mathrm{TEC}}^{U}(t)$ represent the ionospheric influence. Then, we can get the RDSS uplink signal frequency as follows:

$$
f_{\mathrm{RDSS}}^{U}(t)=M_{\mathrm{RDSS}} \times f_{o}^{c}+\Delta f_{1}(t)+\Delta f_{\mathrm{TEC}}^{U}(t)
$$

Similar to the RNSS signal analysis above, if we assume the $C_{\mathrm{RDSS}}$ represents the $1-\mathrm{GHz}$ carrier frequency ionospheric influence factor of RDSS signal and the $k_{\mathrm{RDSS}}$ represents the RDSS relative motion carrier Doppler frequency coefficient, then we can get the following equations:

$$
\begin{aligned}
& C_{\mathrm{RDSS}}=\frac{10^{18}}{f_{\mathrm{RDSS}}^{2}} \\
& k_{\mathrm{RDSS}}=\frac{f_{\mathrm{RDSS}}}{1 \mathrm{GHz}}
\end{aligned}
$$

Then, the RDSS signal frequency the user received can be expressed as follows:

$$
\begin{aligned}
f_{\mathrm{RDSS}}^{r}(t)=\{ & M_{\mathrm{RDSS}} \times f_{o}^{c}+\Delta f_{1}(t)+\Delta f_{\mathrm{TEC}}^{U}(t)-P_{\mathrm{RDSS}} \\
& \times\left(f_{o}+\Delta f_{o}(t)\right)+k_{\mathrm{RDSS}} \times \Delta f_{2}(t)+C_{\mathrm{RDSS}} \\
& \left.\times \Delta f_{\mathrm{TEC}}(t)\right\} \times \frac{f_{o}^{r}}{f_{o}^{r}+\Delta f_{r}(t)}
\end{aligned}
$$

The RDSS signal also has the transmission path from user to MCS, and the transmission path is the same as the path from MCS to user signal. The user received the RDSS signal which is transmitted from MCS to the user and generated signals at the receiver to the MCS via satellite $\mathrm{L}-\mathrm{C}$ transponders. 


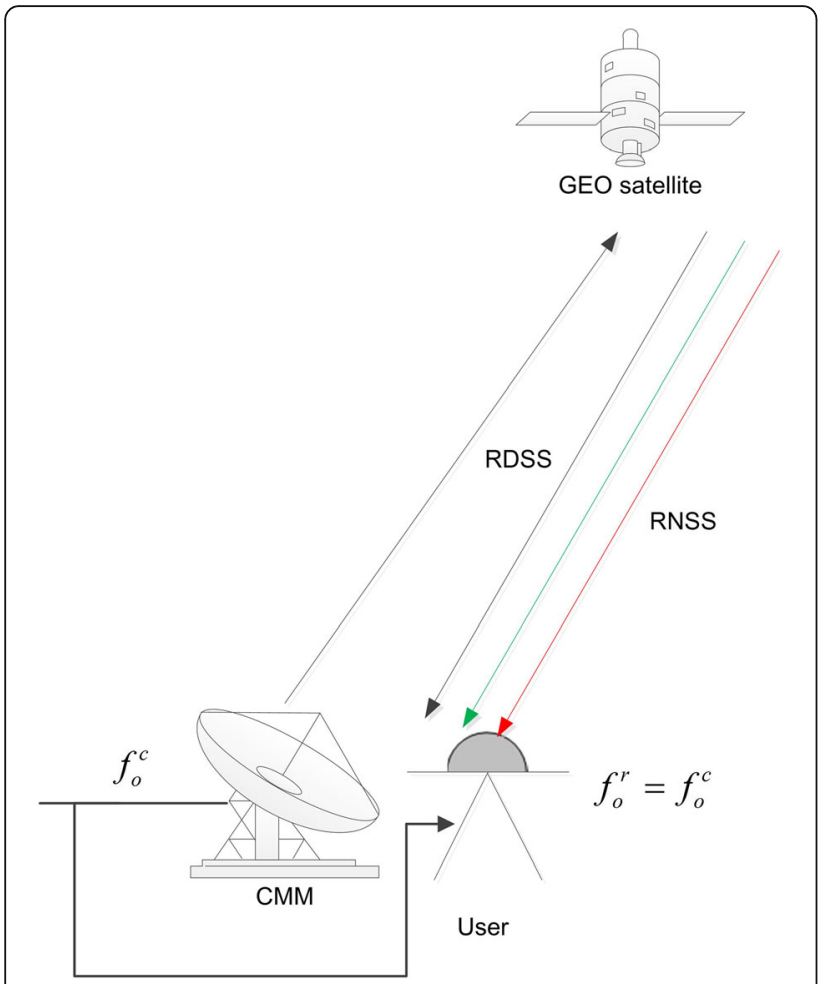

Fig. 3 Precise time and frequency transmission between MCS and satellite

\section{Method: precise time and frequency transfer between MCS and satellite combined RDSS and RNSS signals}

In this section, we discuss the time and frequency synchronization method for MCS and satellites by placing a receiver with the MCS clock source in the MCS. The application of this method is as follows.

In this mode, the user is in the same location as the MCS, and the user receiver and the MCS share the same clock source which is the MCS clock source $f_{o}^{c}$. Therefore, we can get the equation $f_{o}^{r}=f_{o}^{c}, \Delta f_{r}(t)=0$ (as shown in Fig. 3). The RDSS and RNSS signals are measured at the same epoch time, and the carrier phase information of RDSS and RNSS is jointly calculated to obtain ionospheric Doppler $\Delta f_{\mathrm{TEC}}(t)$ (effect of ionosphere on signal carrier frequency), motion Doppler $\Delta f_{2}(t)$ (effect of the relative motion of satellites and user on signal carrier frequency), and satellite clock drift $\Delta f_{o}(t)$ (effect of the satellite clock drift on signal carrier frequency).

In this case, the frequency drift of receiver clock $\Delta f^{r}(t)=$ 0 . We rewrite the RDSS and RNSS carrier frequency observation equations Eq. (7) as follows:

$f_{\mathrm{RNSS}(i)}^{r}(t)=\left\{N_{i}\left[f_{o}+\Delta f_{o}(t)\right]+k_{i} \times \Delta f_{2}(t)+c_{i} \times \Delta f_{\mathrm{TEC}}(t)\right\}$

$$
\begin{aligned}
f_{\mathrm{RDSS}}^{r}(t)= & M_{\mathrm{RDSS}} \times f_{o}^{c}+\Delta f_{1}(t) \\
& +\Delta f_{\mathrm{TEC}}^{U}(t)-P_{\mathrm{RDSS}} \times\left(f_{o}+\Delta f_{o}(t)\right) \\
& +k_{\mathrm{RDSS}} \times \Delta f_{2}(t)+C_{\mathrm{RDSS}} \\
& \times \Delta f_{\mathrm{TEC}}(t)
\end{aligned}
$$

In the equations above, we can see that the $N_{i}, k_{i}$, $f_{o}, c_{i}, f_{o}^{r}, M_{\mathrm{RDSS}}, P_{\mathrm{RDSS}}, k_{\mathrm{RDSS}}$, and $C_{\mathrm{RDSS}}$ can be known from the BeiDou system configuration. Because the MCS and the receiver are in the same position, their RDSS uplink and downlink signal paths overlap. In this case, the ionosphere and Doppler relationship can be obtained as follows:

$$
\begin{aligned}
& \Delta f_{\mathrm{TEC}}^{U}(t)=C_{\mathrm{RDSS}}^{U} \times \Delta f_{\mathrm{TEC}}(t) \\
& \Delta f_{1}(t)=k_{\mathrm{RDSS}}^{U} \times \Delta f_{2}(t)
\end{aligned}
$$

where $C_{\mathrm{RDSS}}^{U}=\frac{10^{18}}{\left(M_{\mathrm{RDSS}} \times f^{c}\right)^{2}}, k_{\mathrm{RDSS}}^{U}=\frac{M_{\mathrm{RDSS} \times f_{o}^{c}}}{1 \mathrm{GHz}}$. The $C_{\mathrm{RDSS}}^{U}$ represents the $1-\mathrm{GHz}$ carrier frequency ionospheric influence factor of RDSS signal; the $k_{\mathrm{RDSS}}^{U}$ represents the RDSS carrier Doppler frequency coefficient. They also can be known from the BeiDou system configuration.

In the joint receiver of BeiDou's RDSS and RNSS (which receive and process RDSS, RNSS with same clock and measured at the same epoch), we can get the RDSS and RNSS signal carrier frequency. By solving the abovementioned RDSS and RNSS frequency equations, we can obtain the solutions of the unknowns in the equations and obtain the satellite atomic clock drift value $\Delta f_{o}(t)$ (there are specific case analyses behind this paper). If only the multifrequency RNSS signal equation is applied, solving the equations is ill-conditioned, and therefore, the satellite clock error cannot be solved.

After obtaining the satellite atomic clock frequency drift value, we can further realize the precision time synchronization of satellites and MCS time by combining the RNSS pseudorange information at the epoch time. Figure 4 shows a block diagram of the time transfer process flowchart, where $\rho_{B i}$ represents the pseudorange measurement of the $B(i)$ signal of the receiver, $\phi_{B i}$ represents the carrier phase measurement of the $\mathrm{B}(\mathrm{i})$ signal of the receiver. As shown in Fig. 4, we can further utilize the pseudorange and carrier phase observations of multi-frequency RNSS signals to achieve time transmission between MCS and satellite.

\subsection{Calculation of Doppler and satellite clock errors using RDSS and one RNSS observation}

When the ionosphere TEC on the path of signal propagation is known in advance, the unknown variables to solve are $\Delta f_{2}(t)$ (carrier Doppler caused by the relative movement of satellites and receivers) and 
$\Delta f_{o}(t)$ (satellite clock drift). In this case, we can use RDSS and one RNSS observation to get the satellite clock drift value. When the ionosphere TEC is known, we mark the $\Delta \operatorname{TEC}_{i}(t)=c_{i} \times \Delta f_{\mathrm{TEC}}(t)$, which is a known value. Referencing Eq. (12), we can get the following equations:

$$
f_{\mathrm{RNSS}(i)}^{r}(t)=\left\{N_{i}\left[f_{o}+\Delta f_{o}(t)\right]+k_{i} \times \Delta f_{2}(t)+c_{i} \times \Delta f_{\mathrm{TEC}}(t)\right\}
$$

$$
\begin{aligned}
=\left\{N_{i} f_{o}\right. & +N_{i} \Delta f_{o}(t)+\frac{N_{i} f_{o}}{1 \mathrm{GHz}} \times \Delta f_{2}(t)+\frac{N_{i}}{1 \mathrm{GHz}} \\
& \left.\times \Delta f_{o}(t) \times \Delta f_{2}(t)+\Delta \mathrm{TEC}_{i}(t)\right\}
\end{aligned}
$$

We can see that there is a nonlinear term in Eq. (16), which is the product of the unknown quantity $\Delta f_{o}(t)$ and $\Delta f_{2}(t)$ to be solved. It is as follows:

$$
\frac{N_{i}}{1 \mathrm{GHz}} \times \Delta f_{o}(t) \times \Delta f_{2}(t)
$$

By analyzing the coefficient of this item, we can see that its coefficient level is about $10^{-7}$ orders, and the accuracy of the satellite clock is much lower than this magnitude, so we can completely ignore this crossproduct term in our recalculation. Thus, Eq. (16) can be simplified as follows:

$$
\begin{aligned}
f_{\mathrm{RNSS}(i)}^{r}(t)= & N_{i} f_{o}+N_{i} \Delta f_{o}(t)+\frac{N_{i} f}{1 \mathrm{GHz}} \\
& \times \Delta f_{2}(t)+\Delta \operatorname{TEC}_{i}(t)
\end{aligned}
$$

For RDSS signals, similar to the RNSS signal analysis, the following equation can be obtained by appropriate simplification:

$$
\begin{aligned}
f_{\mathrm{RDSS}}^{r}(t)= & P_{\mathrm{RDSS}} \Delta f_{o}(t) \\
& +\left[\frac{2 M_{\mathrm{RDSS}} f_{o}^{c}+P_{\mathrm{RDSS}} f_{o}(t)}{1 G H z}\right] \Delta f_{2}(t) \\
& +\left\{\left[M_{\mathrm{RDSS}} f_{o}^{c}+P_{\mathrm{RDSS}} f_{o}\right]+\Delta \mathrm{TEC} 2\right\}
\end{aligned}
$$

where $\Delta \mathrm{TEC} 2=\Delta f_{\mathrm{TEC}}^{U}(t)+C_{\mathrm{RDSS}} \times \Delta f_{\mathrm{TEC}}(t)$; it is the ionosphere TEC influence on the whole RDSS signal carrier frequency.

So we can select one RNSS observation which is shown in Eq. (18) and RDSS observation which is shown in Eq. (19) to solve unknown variables $\Delta f_{o}(t)$ and $\Delta f_{2}(t)$ together; the solution equation in matrix form can be written as shown below:

$$
\begin{aligned}
{\left[\begin{array}{c}
f_{\mathrm{RNSS}(i)}^{r}(t) \\
f_{\mathrm{RDSS}}^{r}(t)
\end{array}\right]=} & {\left[\begin{array}{cc}
N_{i} & \frac{N_{i} f}{1 \mathrm{GHz}} \\
P_{\mathrm{RDSS}} & \frac{2 M_{\mathrm{RDSS}} f_{o}^{c}+P_{\mathrm{RDSS}} f_{o}(t)}{1 \mathrm{GHz}}
\end{array}\right] } \\
& \times\left[\begin{array}{c}
\Delta f_{o}(t) \\
\Delta f_{2}(t)
\end{array}\right] \\
& +\left[\begin{array}{c}
N_{i} f_{o}+\Delta \mathrm{TEC}_{i}(t) \\
{\left[M_{\mathrm{RDSS}} f_{o}^{c}+P_{\mathrm{RDSS}} f_{o}\right]+\Delta \mathrm{TEC} 2}
\end{array}\right]
\end{aligned}
$$

In the above equation, the meaning of the letter is the same as the above article. The unknowns with the solution are $\Delta f_{o}(t)$ and $\Delta f_{2}(t)$; the $f_{\mathrm{RNSS}(i)}^{r}(t)$ and $f_{\mathrm{RDSS}}^{r}(t)$ can be got by the carrier phase observations of the receiver. Others are either known quantities that the system configuration can determine or known quantities obtained by measurement.

But we cannot use two RNSS observations to calculate the Doppler and satellite clock errors. Although both RNSS observation equations contain $\Delta f_{o}(t)$ and $\Delta f_{2}(t)$ unknowns, the solution equation in matrix form can be written as shown below. In this case, the equations are parallel, forming ill-conditioned equations:

$$
\left[\begin{array}{l}
f_{\mathrm{RNS}(1)}^{r}(t) \\
f_{\mathrm{RNSS}(2)}^{r}(t)
\end{array}\right]=\left[\begin{array}{ll}
N_{1} & \frac{N_{1} f}{1 \mathrm{GHz}} \\
N_{2} & \frac{N_{2} f}{1 \mathrm{GHz}}
\end{array}\right]\left[\begin{array}{l}
\Delta f_{o}(t) \\
\Delta f_{2}(t)
\end{array}\right]+\left[\begin{array}{l}
N_{1} f_{o}+\Delta \operatorname{TEC}_{1}(t) \\
N_{2} f_{o}+\Delta \operatorname{TEC}_{2}(t)
\end{array}\right]
$$

The meaning of the letter is the same as Eq. (18). The coefficient matrix before the unknown is to be solved is not full rank.

\subsection{Calculation of Doppler, satellite clock, and ionospheric carrier Doppler using one RDSS and two RNSS observations}

When the ionosphere TEC on the satellite to the MCS signal propagation path is unknown, the unknown variables to solve become three which are $\Delta f_{2}(t), \Delta f_{o}(t)$, and $\Delta f_{\text {TEC }}(t)$. In this case, we can use RDSS and two RNSS observations to get the satellite clock drift value.

Similar to the analysis in Section 3.1, we derive the RDSS and RNSS carrier frequency observation equations as follows:

$$
\begin{aligned}
f_{\mathrm{RNSS}(i)}^{r}(t)=\{ & N_{i}\left[f_{o}+\Delta f_{o}(t)\right]+k_{i} \times \Delta f_{2}(t)+c_{i} \\
& \left.\times \Delta f_{\mathrm{TEC}}(t)\right\}=N_{i} \Delta f_{o}(t)+\frac{N_{i} f_{o}}{1 \mathrm{GHz}} \\
& \times \Delta f_{2}(t)+c_{i} \times \Delta f_{\mathrm{TEC}}(t)+N_{i} f_{o}
\end{aligned}
$$




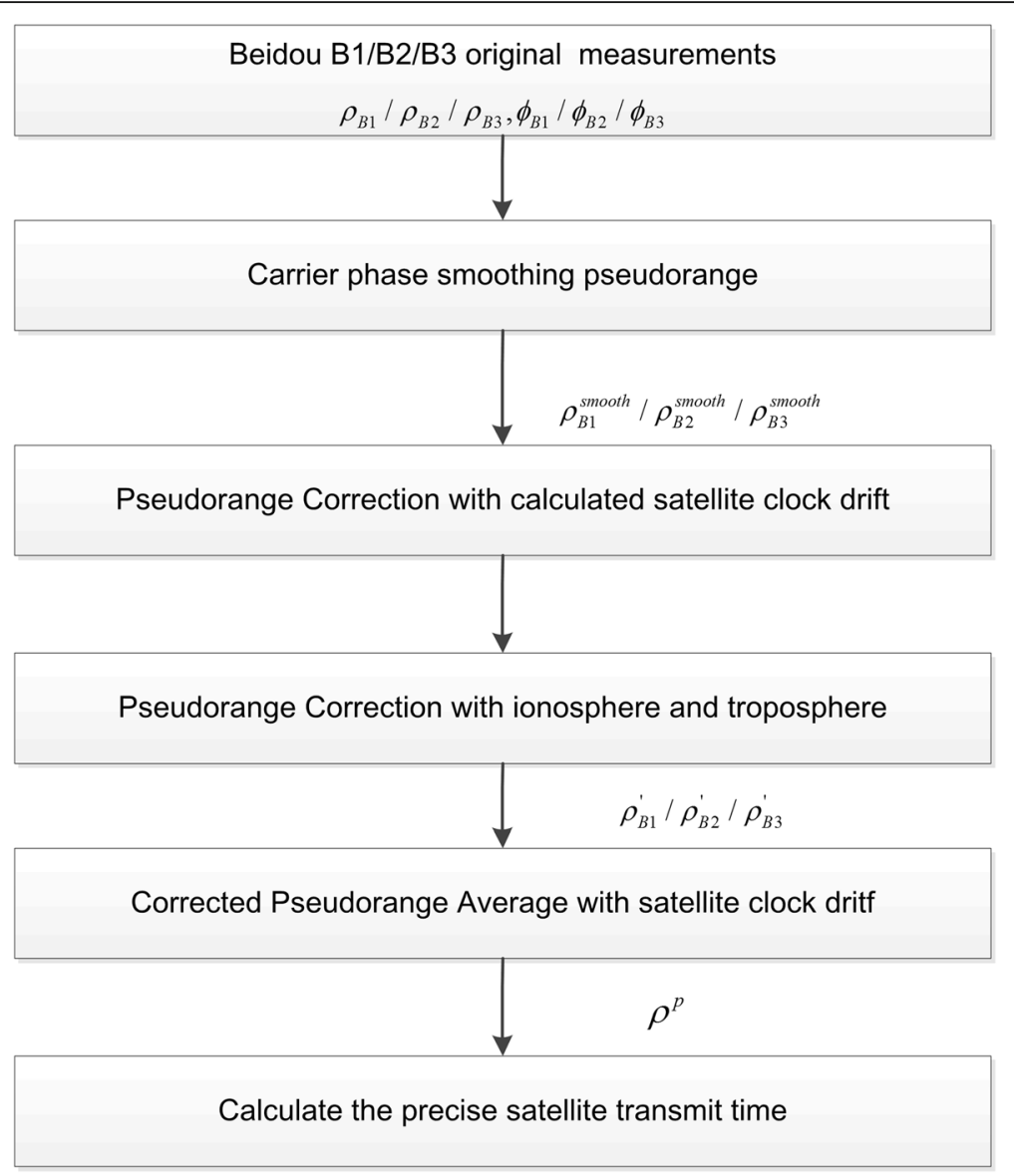

Fig. 4 Block diagram of the time transfer process flowchart between MCS and satellite

$$
\begin{aligned}
f_{\mathrm{RDSS}}^{r}(t)=\left\{M_{\mathrm{RDSS}}\right. & \times f_{o}^{c}+\Delta f_{1}(t)+\Delta f_{\mathrm{TEC}}^{U}(t)-P_{\mathrm{RDSS}} \\
& \times\left(f_{o}+\Delta f_{o}(t)\right)+k_{\mathrm{RDSS}} \times \Delta f_{2}(t) \\
& \left.+C_{\mathrm{RDSS}} \times \Delta f_{\mathrm{TEC}}(t)\right\}=P_{\mathrm{RDSS}} \Delta f_{o}(t) \\
& +\left[\frac{2 M_{\mathrm{RDSS}} f_{o}^{c}+P_{\mathrm{RDSS}} \times f_{o}}{1 \mathrm{GHz}}\right] \Delta f_{2}(t) \\
& +C_{\mathrm{RDSS}} \times \Delta f_{\mathrm{TEC}}(t) \\
& +\left[M_{\mathrm{RDSS}} \times f_{o}^{c}+\mathrm{RDSS} \times f_{o}\right]
\end{aligned}
$$

So we can select two RNSS observation which is shown in Eq. (22) and RDSS observation which is shown in Eq. (23) to solve unknown variables $\Delta f_{o}(t)$, $\Delta f_{2}(t)$, and $\Delta f_{T E C}(t)$ together. Then, $\Delta f_{o}(t)$ can be used to synchronize the clock of satellite and MCS. The solution equation in matrix form can be written as shown below:

$$
\begin{aligned}
& {\left[\begin{array}{c}
f_{\mathrm{RNSS}(1)}^{r}(t) \\
f_{\mathrm{RNS}(2)}^{r}(t) \\
f_{\mathrm{RDSS}}^{r}(t)
\end{array}\right]=\left[\begin{array}{ccc}
N_{1} & \frac{N_{1} f_{o}}{1 \mathrm{GHz}} & c_{1} \\
N_{2} & \frac{N_{2} f_{o}}{1 \mathrm{GHz}} & c_{2} \\
& \frac{2 M_{\mathrm{RDSS}} f_{o}^{c}+P_{\mathrm{RDSS}} \times f_{o}}{1 \mathrm{GHz}} & C_{\mathrm{RDSS}}
\end{array}\right]} \\
& {\left[\begin{array}{c}
\Delta f_{o}(t) \\
\Delta f_{2}(t) \\
\Delta f_{\mathrm{TEC}}(t)
\end{array}\right]+\left[\begin{array}{c}
N_{1} f_{o} \\
N_{2} f_{o} \\
M_{\mathrm{RDSS}} \times f_{o}^{c}+P_{\mathrm{RDSS}} \times f_{o}
\end{array}\right]}
\end{aligned}
$$

In the above equation, the meaning of the letter is the same as the above article. The unknowns with the solution are $\Delta f_{o}(t), \Delta f_{2}(t)$, and $\Delta f_{\mathrm{TEC}}(t)$; the $f_{\mathrm{RNSS}(1)}^{r}(t), f_{\mathrm{RNSS}(2)}^{r}(t)$, and $f_{\mathrm{RDSS}}^{r}(t)$ can be got by the carrier phase observations of the receiver. Others are either known quantities that the system configuration can determine or known quantities obtained by measurement. 


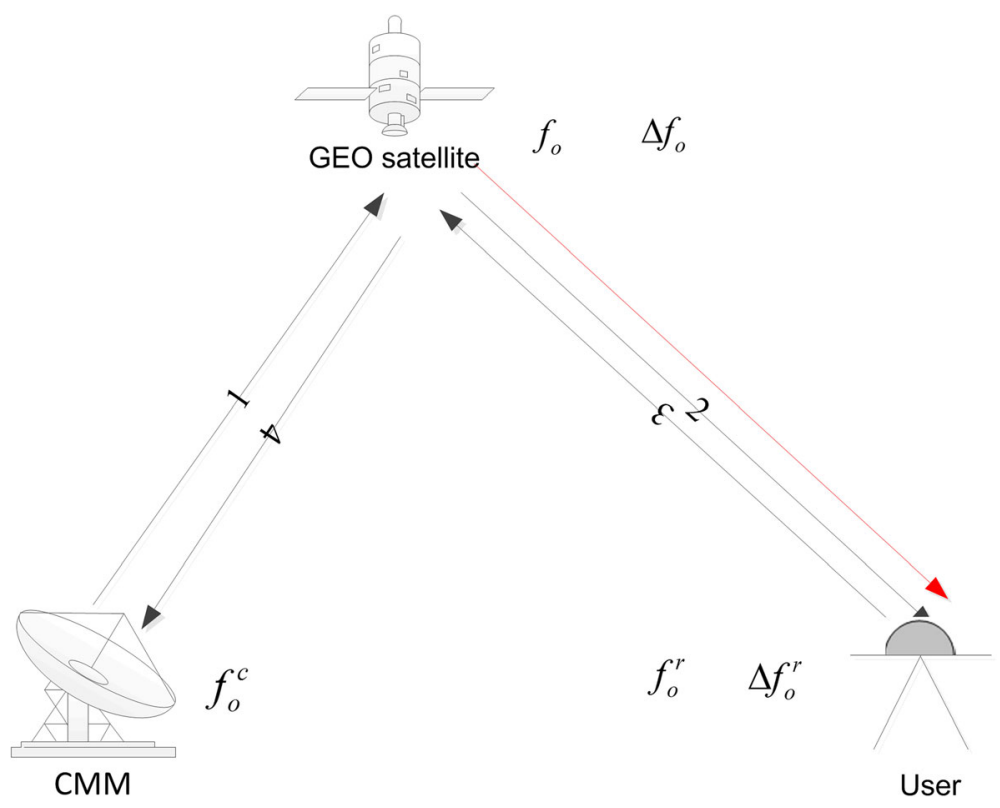

Fig. 5 Precise time and frequency transmission between MCS and user

\section{Method: precise time and frequency transfer between MCS and user combined RDSS and RNSS signals}

In this section, we discuss the time and frequency transfer method for MCS and user by jointly using RDSS and RNSS observations. This method uses the joint calculation of RDSS and RNSS signals. The application of this method is as follows.

In this mode, the location of the user is different from the MCS, the MCS and users have their own clock source, and the RDSS signals have different propagation paths. As shown in Fig. 5, paths 1 and 4 of the RDSS signals which are between MCS and satellite have the same propagation paths, and paths 2 and 3 of the RDSS signals which are between user and satellite have other same propagation paths. This is different from the case above. However, we can see that paths 2 and 3 of the RDSS signals have the same propagation paths as the RNSS signals.

In this case, we can measure the carrier phase information of the RDSS signal at the MCS and the user, respectively, and use the RDSS and RNSS carrier phase observations to jointly calculate the Doppler value $\Delta f_{2}(t)$, satellite clock drift $\left(\Delta f_{o}(t)\right)$, and user crystal frequency $\operatorname{drift}\left(\Delta f_{o}^{r}(t)\right)$.

Similar to the previous analysis, we first establish a measurement equation for the RDSS signal. As shown in the previous Eqs. (7) and (11), the RDSS signal carrier frequency value measured by the user can be written as follows:

$$
\begin{aligned}
& f_{\mathrm{RDSS}}^{r}(t)=\left\{\begin{array}{l}
M_{\mathrm{RDSS}} \times f_{o}^{c}+\Delta f_{1}(t)+\Delta f_{\mathrm{TEC}}^{U}(t)-P_{\mathrm{RDSS}} \times\left(f_{o}\right. \\
\left.+\Delta f_{o}(t)\right)+k_{\mathrm{RDSS}} \times \Delta f_{2}(t)+C_{\mathrm{RDSS}} \times \Delta f_{\mathrm{TEC}}(t)
\end{array}\right\} \\
& \times \frac{f_{o}^{r}}{f_{o}^{r}+\Delta f_{r}(t)}
\end{aligned}
$$

The RNSS signal carrier frequency value measured by the user can be written as follows:

$$
\begin{aligned}
f_{\mathrm{RNSS}(i)}^{r}(t)=\left\{N _ { i } \left[f_{o}\right.\right. & \left.\left.+\Delta f_{o}(t)\right]+k_{i} \times \Delta f_{2}(t)+c_{i} \times \Delta f_{\mathrm{TEC}}(t)\right\} \\
& \times \frac{f_{o}^{r}}{f_{o}^{r}+\Delta f_{r}(t)}
\end{aligned}
$$

We can see that different from RNSS observation, the RDSS observation contains the MCS standard clock $f_{o}^{c}$. The RDSS signal received by the user and the user generated a fixed frequency signal which was forwarded to the MCS via the satellite so that the MCS received user backhaul RDSS signal. Let us assume that the local oscillator of path 3 of the RDSS signal generated by the user is $f_{\mathrm{LO}(\text { (ideal) }}^{\text {user }}$, and its relationship with the receiver crystal oscillator frequency is as follows:

$$
f_{\mathrm{LO}(\text { ideal })}^{\mathrm{user}}=N_{\mathrm{LO}}^{\mathrm{user}} \times f_{o}^{r}
$$

where the $N_{\mathrm{LO}}^{\text {user }}$ represents the multiple of the signal frequency of path 3 of the RDSS signal generated by the 
user and user oscillator signal $f_{o}^{r}$. When the local frequency of the receiver is determined, the $N_{\mathrm{LO}}^{\mathrm{user}}$ is also a definite value.

At this time, the receiver's actual generated local oscillator signal is affected by the receiver crystal oscillator drift, as shown in the following formula:

$$
f_{\mathrm{LO}}^{\text {user }}(t)=N_{\mathrm{LO}}^{\mathrm{user}} \times\left(f_{o}^{r}+\Delta f_{r}(t)\right)
$$

Also, let us assume that the local oscillator of the satellite which is used to forward users to MCS signals is $f_{\mathrm{LO}}^{\text {sat }}$, and its relationship with the satellite clock is as follows:

$$
f_{\mathrm{LO}(\text { ideal })}^{\text {sat }}=N_{\mathrm{LO}}^{\mathrm{sat}} \times f_{o}
$$

where $N_{\mathrm{LO}}^{\mathrm{sat}}$ is also a deterministic value in the BeiDou's system.

The satellite's real generated local oscillator signal is affected by the satellite atomic clock drift, as shown in the following formula:

$$
f_{\mathrm{LO}}^{\mathrm{sat}}(t)=N_{\mathrm{LO}}^{\mathrm{sat}} \times\left(f_{o}+\Delta f_{o}(t)\right)
$$

The expression of the signal sent by the user to the MCS at the satellite receiving end is as follows:

$$
f_{\mathrm{RDSS}}^{u U}(t)=f_{\mathrm{LO}}^{\mathrm{user}}+\frac{10^{18}}{\left(f_{\mathrm{LO}}^{\mathrm{user}}\right)^{2}} \Delta f_{\mathrm{TEC}}(t)+\frac{f_{\mathrm{LO}}^{\mathrm{user}}}{1 \mathrm{GHz}} \Delta f_{2}(t)
$$

The expression of the RDSS signal frequency received by the MCS which is transmitted from the user to the MCS is as follows:

$$
f_{\mathrm{RDSS}}^{C D}(t)=f_{\mathrm{RDSS}}^{U U}(t)+f_{\mathrm{LO}}^{\mathrm{sat}}(t)+\Delta \mathrm{TEC} 2+\Delta f_{2}^{\prime}(t)
$$

where the $\triangle \mathrm{TEC} 2$ represents the carrier frequency due to the influence of ionospheric on path 4 of the RDSS, and the $\Delta f_{2}^{\prime}$ represents the carrier Doppler frequency duo to relative motion of satellites and MCS. Also, when the ionospheric TEC on the path between MCS and satellite is known, and relative motion of satellites and MCS is known, the $\triangle T E C 2$ and $\triangle f_{2}^{\prime}$ can be obtained by simple mathematical calculation.
Then, by solving equations composed of Eq. (25), Eq. (26), and Eq. (32), we can solve the $\Delta f_{o}(t), \Delta f_{2}(t)$, and $\Delta f_{r}(t)$ three unknown variables. This requires that the ionosphere and motion Doppler influence information on 1 and 4 signal propagation paths of the RDSS be known, which was already presented in the second part of the paper.

Simplify Eqs. (25), (26), and (32) to get the following equations:

$$
\begin{aligned}
f_{\mathrm{RDSS}}^{r}(t)=\left\{P_{\mathrm{RDSS}} \Delta f_{o}(t)+\left[\frac{2 M_{\mathrm{RDSS}} f_{o}^{c}+P_{\mathrm{RDSS}} \times f_{o}}{1 \mathrm{GHz}}\right] \Delta f_{2}(t)\right. \\
\left.+C_{\mathrm{RDSS}} \times \Delta f_{\mathrm{TEC}}(t)+\left[M_{\mathrm{RDSS}} \times f_{o}^{c}+\mathrm{RDSS} \times f_{o}\right]\right\} \\
\times \frac{f_{o}^{r}}{f_{o}^{r}+\Delta f_{r}(t)} \\
f_{\mathrm{RNSS}(i)}^{r}(t)=\left\{N_{i} f_{o}+N_{i} \Delta f_{o}(t)+\frac{N_{i} f}{1 \mathrm{GHz}} \times \Delta f_{2}(t)\right. \\
\left.+\Delta \mathrm{TEC}_{i}(t)\right\} \times \frac{f_{o}^{r}}{f_{o}^{r}+\Delta f_{r}(t)} \\
f_{\mathrm{RDSS}}^{C D}(t)= \\
\quad N_{\mathrm{LO}}^{\mathrm{sat}} \times \Delta f_{o}(t)+N_{\mathrm{LO}}^{\mathrm{user}} \times \Delta f_{r}(t) \\
+\frac{10^{18}}{\left(N_{\mathrm{LO}}^{\mathrm{user}} f_{o}^{r}\right)^{2}} \Delta f_{\mathrm{TEC}}(t) \\
+\frac{N_{\mathrm{LO}}^{\mathrm{user}} f_{o}^{r}}{1 \mathrm{GHz}} \Delta f_{2}(t)+\Delta f_{2}^{\prime}(t) \\
+\Delta \mathrm{TEC} 2+N_{\mathrm{LO}}^{\mathrm{user}} f_{o}^{r}+N_{\mathrm{LO}}^{\mathrm{sat}} f_{o}
\end{aligned}
$$

Since the $\Delta f_{o}(t)$ and $\Delta f_{2}(t)$ cannot be distinguished by only RNSS observations, when we use the three RNSS signal equations to solve the three unknown variables $\Delta f_{o}(t), \Delta f_{2}(t)$, and $\Delta f_{r}(t)$, we will form illconditioned equations. So, we must use RDSS observation at receiver and user backhaul RDSS signal to establish another equation in the observation of the MCS. By solving the equations, we obtain the frequency difference information between the receiver and the MCS standard clock, and adjust the receiver clock through this frequency difference information to achieve high-precision frequency at the receiver.

Similar to the above, we can get $\Delta f_{r}(t)$ which is the deviation of the receiver crystal oscillator, and the system of solution equations formed by Eqs. (33), (34), and (35) can be written as shown below:

$$
\left\{\begin{array}{c}
f_{\mathrm{RNSS}(i)}^{r}(t)=\left\{N_{i} f_{o}+N_{i} \Delta f_{o}(t)+\frac{N_{i} f}{1 \mathrm{GHz}} \times \Delta f_{2}(t)+\Delta \mathrm{TEC}_{i}(t)\right\} \times \frac{f_{o}^{r}}{f_{o}^{r}+\Delta f_{r}(t)} \\
f_{\mathrm{RDSS}}^{r}(t)=\left\{P_{\mathrm{RDSS}} \Delta f_{o}(t)+\left[\frac{2 M_{\mathrm{RDSS}} f_{o}^{c}+P_{\mathrm{RDSS}} \times f_{o}}{1 \mathrm{GHz}}\right] \Delta f_{2}(t)+C_{\mathrm{RDSS}} \times \Delta f_{\mathrm{TEC}}(t)+\left[M_{\mathrm{RDSS}} \times f_{o}^{c}+\mathrm{RDSS} \times f_{o}\right]\right\} \times \frac{f_{o}^{r}}{f_{o}^{r}+\Delta f_{r}(t)} \\
f_{\mathrm{RDSS}}^{C D}(t)=N_{\mathrm{LO}}^{\mathrm{sat}} \times \Delta f_{o}(t)+N_{\mathrm{LO}}^{\mathrm{user}} \times \Delta f_{r}(t)+\frac{10^{18}}{\left(N_{\mathrm{LO}}^{\mathrm{user}} f_{o}^{r}\right)^{2}} \Delta f_{\mathrm{TEC}}(t)+\frac{N_{\mathrm{LO}}^{\mathrm{user}} f_{o}^{r}}{1 \mathrm{GHz}} \Delta f_{2}(t)+\Delta f_{2}^{\prime}(t)+\Delta \mathrm{TEC} 2+N_{\mathrm{LO}}^{\mathrm{user}} f_{o}^{r}+N_{\mathrm{LO}}^{\text {sot }} f_{o}
\end{array}\right.
$$




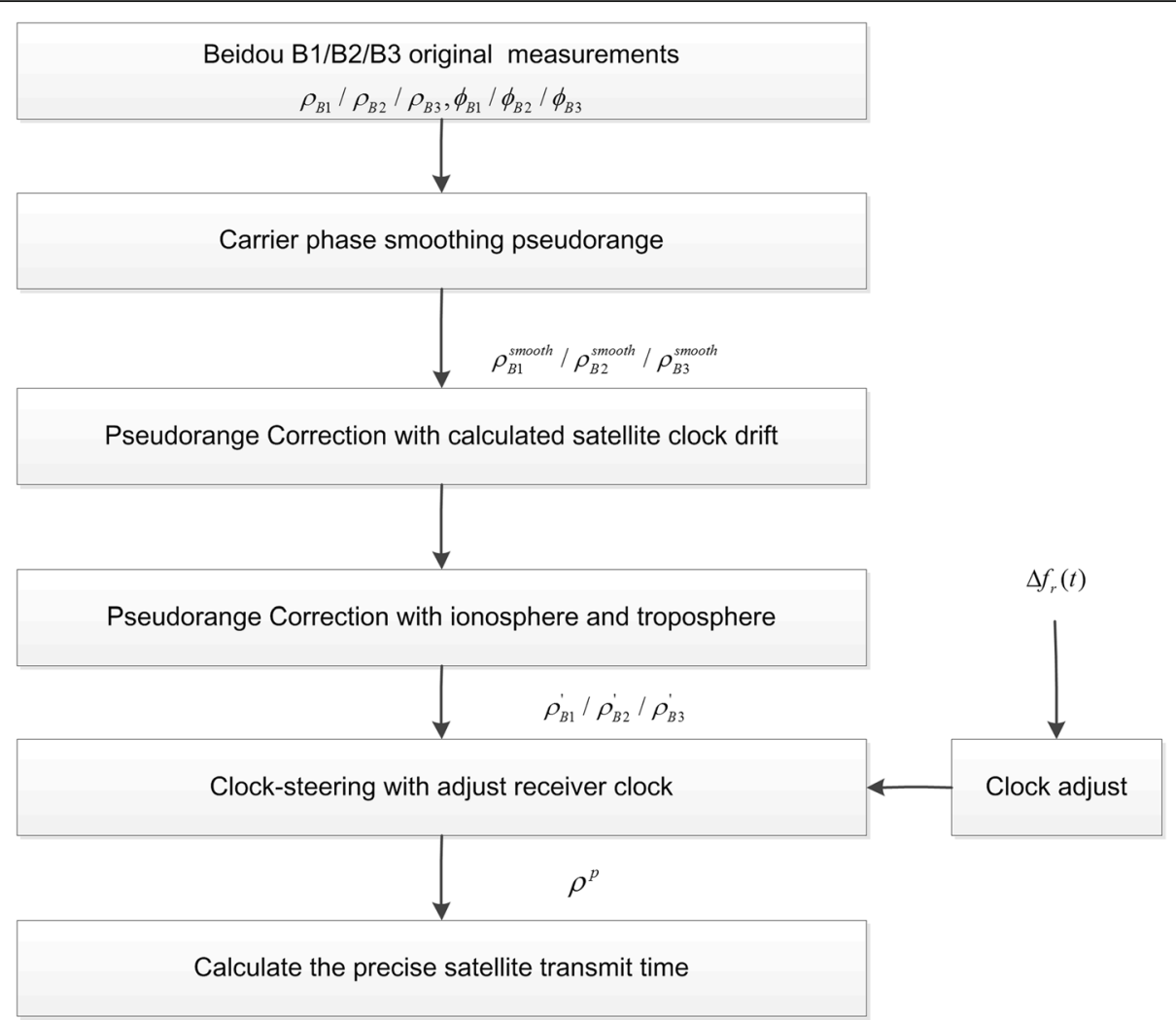

Fig. 6 Block diagram of the time transfer process flowchart between MCS and user

It can be seen that the system of equations is a system of quadratic equations involving the product of unknown variables. The solution of the unknowns requires further mathematical transformations to be implemented. The unknown variables with the solution are $\Delta f_{o}(t), \Delta f_{2}(t)$, and $\Delta f_{\mathrm{TEC}}(t)$; the $f_{\mathrm{RNSS}(i)}^{r}(t), f_{\mathrm{RDSS}}^{r}(t)$ and $f_{\mathrm{RDSS}}^{C D}(t)$ can be got by the carrier phase observations of the receiver and the MCS. Others are either known quantities that the system configuration can determine or known quantities obtained by measurement.

Figure 6 shows the block diagram of the time transfer process flowchart, where $\rho_{B i}$ represents the pseudorange measurement of the $B(i)$ signal of the receiver, and $\phi_{B i}$ represents the carrier phase measurement of the $B(i)$ signal of the receiver. As shown in Fig. 6, we can further utilize the pseudorange and carrier phase observations of multi-frequency RNSS signals to achieve time transmission between CMM and user.

\section{Discussion and experiments}

In this section, we discuss the time and frequency transfer error of the method which is jointly implemented by RDSS and RNSS as described in the paper and carry out relevant computer simulation verification.

\subsection{Error analysis}

As described in the third and fourth part of the paper, the algorithm of this paper combined the BeiDou's RDSS and RNSS signals to realize the solving equation of precise time and frequency transmission between MCS and satellite and between MCS and user.

In this paper, by simplifying the related equations, the linear combination of RDSS and RNSS frequency observations and related unknowns is obtained. In this case, the error of each unknown variables to be solved based on this observations can be obtained through a simple mathematical transformation.

The following uses Section 3.2 of the paper as an example to illustrate the error solution process of the algorithm. In this case, the RDSS and RNSS carrier frequency observation equations are as Eqs. (22) and (23). If we use B1, B2, and RDSS signal to jointly solve $\Delta f_{2}(t), \Delta f_{o}(t)$, and $\Delta f_{T E C}(t)$, then the $\mathrm{B} 1, \mathrm{~B} 2$, and RDSS carrier frequency observation equations are as follows: 


$$
\left\{\begin{array}{c}
f_{\mathrm{RNSS}(1)}^{r}(t)==N_{1} \Delta f_{o}(t)+\frac{N_{1} f_{o}}{1 \mathrm{GHz}} \times \Delta f_{2}(t)+c_{1} \times \Delta f_{\mathrm{TEC}}(t)+N_{1} f_{o} \\
f_{\mathrm{RNSS}(2)}^{r}(t)==N_{2} \Delta f_{o}(t)+\frac{N_{2} f_{o}}{1 \mathrm{GHz}} \times \Delta f_{2}(t)+c_{2} \times \Delta f_{\mathrm{TEC}}(t)+N_{2} f_{o} \\
f_{\mathrm{RDSS}}^{r}(t)=P_{\mathrm{RDSS}} \Delta f_{o}(t)+\left[\frac{2 M_{\mathrm{RDSS}} f_{o}^{c}+P_{\mathrm{RDSS}} \times f_{o}}{1 \mathrm{GHz}}\right] \Delta f_{2}(t)+C_{\mathrm{RDSS}} \times \Delta f_{\mathrm{TEC}}(t)+\left[M_{\mathrm{RDSS}} \times f_{o}^{c}+P_{\mathrm{RDSS}} \times f_{o}\right]
\end{array}\right.
$$

Convert to matrix form as follows:

$$
\begin{aligned}
{\left[\begin{array}{c}
f_{\mathrm{RNSS}(1)}^{r}(t) \\
f_{\mathrm{R} N(2)}^{\mathrm{R}(t)}(t) \\
f_{\mathrm{RDSS}}^{\prime \prime}(t)
\end{array}\right] } & {\left[\begin{array}{ccc}
N_{1} & \frac{N_{1} f_{o}}{1 G \mathrm{HHz}} & c_{1} \\
N_{2} & \frac{N_{2} f_{o}}{16 \mathrm{~Hz}} & (37) c_{2} \\
P_{\mathrm{RDSS}} & \frac{2 M_{\mathrm{RDSS}} f_{o}^{c}+P_{\mathrm{RDSS}} \times f_{o}}{1 \mathrm{GHz}} & C_{\mathrm{RDSS}}
\end{array}\right]\left[\begin{array}{c}
\Delta f_{o}(t) \\
\Delta f_{2}(t) \\
\Delta f_{\mathrm{TEC}}(t)
\end{array}\right] } \\
& +\left[\begin{array}{c}
N_{1} f_{o} \\
N_{2} f_{o} \\
M_{\mathrm{RDSS}} \times f_{o}^{c}+P_{\mathrm{RDSS}} \times f_{o}
\end{array}\right]
\end{aligned}
$$

In Eq. (37), the $f_{\mathrm{RNSS}(1)}^{r}(t), f_{\mathrm{RNSS}(2)}^{r}(t)$, and $f_{\mathrm{RDSS}}^{r}(t)$ are receiver observations, and the $\Delta f_{2}(t), \Delta f_{o}(t)$, and $\Delta f_{\text {TEC }}(t)$ are the unknown variables to be solved. The equation can be further abbreviated as follows:

$$
Y=A X+B
$$

where $Y, A, X, B$ respectively indicate the following:

$$
\begin{aligned}
Y & =\left[\begin{array}{c}
f_{\mathrm{RNSS}(1)}^{r}(t) \\
f_{\mathrm{RNSS}(2)}^{r}(t) \\
f_{\mathrm{RDSS}}^{r}(t)
\end{array}\right] A \\
& =\left[\begin{array}{ccc}
N_{1} & \frac{N_{1} f_{o}}{1 \mathrm{GHz}} & c_{1} \\
N_{2} & \frac{N_{2} f_{o}}{1 \mathrm{GHz}} & c_{2} \\
P_{\mathrm{RDSS}} & \frac{2 M_{\mathrm{RDSS}} f_{o}^{c}+P_{\mathrm{RDSS}} \times f_{o}}{1 \mathrm{GHz}} & C_{\mathrm{RDSS}}
\end{array}\right] X \\
& =\left[\begin{array}{c}
\Delta f_{o}(t) \\
\Delta f_{2}(t) \\
\Delta f_{\mathrm{TEC}}(t)
\end{array}\right] \quad B=\left[\begin{array}{cc}
N_{1} f_{o} \\
M_{\mathrm{RDSS}} \times f_{o}^{c}+P_{\mathrm{RDSS}} \times f_{o}
\end{array}\right]
\end{aligned}
$$

Denote the measurement noise of $f_{\mathrm{RNSS}(1)}^{r}(t), f_{\mathrm{RNSS}(2)}^{r}(t)$, and $f_{\mathrm{RDSS}}^{r}(t)$ by $\varepsilon_{\phi 1}, \varepsilon_{\phi 2}$, and $\varepsilon_{\phi 3} ; \varepsilon_{f}$ represents a matrix consisting of these three values, which were caused by the receiver measurement; denote the $\varepsilon_{\triangle f_{o}}, \varepsilon_{\triangle f_{2}}$, and $\varepsilon_{\triangle f_{T E C}}$ represent the solution error of the unknown variables to be solved which were due to the receiver measurement error; $\boldsymbol{\varepsilon}_{\phi}$ represents a matrix consisting of these three values. From the above formula, we can derive the following formula:

$$
\boldsymbol{\varepsilon}_{f}=\left[\begin{array}{c}
\varepsilon_{\Delta f_{o}} \\
\varepsilon_{\Delta f_{2}} \\
\varepsilon_{\Delta f_{\mathrm{TEC}}}
\end{array}\right] \quad \boldsymbol{\varepsilon}_{\phi}=\left[\begin{array}{c}
\varepsilon_{\phi 1} \\
\varepsilon_{\phi 2} \\
\varepsilon_{\phi 3}
\end{array}\right]
$$

Let $Y^{\prime}$ represent the real measured value matrix of the carrier frequency and let $X^{\prime}$ represent the real measured value matrix of the carrier frequency, then the following formula can be obtained:

$$
Y^{\prime}=Y+\boldsymbol{\varepsilon}_{f}, X^{\prime}=X+\boldsymbol{\varepsilon}_{\phi}
$$

There is the following equation:

$$
\begin{aligned}
& Y^{\prime}=A X^{\prime}+B \\
& Y+\boldsymbol{\varepsilon}_{f}=A\left(X+\boldsymbol{\varepsilon}_{\phi}\right)+B
\end{aligned}
$$

Combining Eqs. (38) and (40), there are the following equations:

$$
\left[\begin{array}{c}
\varepsilon_{\Delta f_{o}} \\
\varepsilon_{\Delta f_{2}} \\
\varepsilon_{\Delta f_{\mathrm{TEC}}}
\end{array}\right]=A^{-1}\left[\begin{array}{c}
\varepsilon_{\phi 1} \\
\varepsilon_{\phi 2} \\
\varepsilon_{\phi 3}
\end{array}\right]
$$

Equation (41) shows the relationship between measurement error and error of solution variables. It can be seen that the solution error of the unknown variables is closely related to the observed noise through the inverse matrix of the equation matrix $\left(A^{-1}\right) ; A$ is determined by the specific configuration of the using RNSS and RDSS signals. It also can be seen that if carrier phase observation errors at different frequencies are independent of each other, the distribution obeys the normal distribution, as the error with the solution variable is a linear combination of the frequency errors and still follows the normal distribution.

The linearization of the solution equations in Section 3.2 of the paper can be written as the above Formula (41). Therefore, for the error analysis under other conditions in the paper, similar to the above analysis, the error conditions for solving unknown variables can be obtained. The only difference is the parameter matrix.

\subsection{Algorithm verification}

In order to verify the correctness and performance of the method in the paper, we simulated the method proposed in the paper. Because the paper simplifies the observation equations to simplify the computation, the ideal error-free solution may produce noise error. The simulation results under no-noise and noisy conditions are given in this paper to verify the performance of the 
simplified algorithm equation and algorithm error conditions in the case of noise.

The signal frequency curve set in the simulation is as shown in the following formula:

(1) The curve of the satellite clock frequency drift $\Delta f_{o}(t)$

We set the frequency curve of the satellite clock as shown in the following formula:

$$
\Delta f_{o}(t)=0.006 \times \sin \left(2 \pi \frac{t}{5000}\right)
$$

where $t=1,2 \ldots 5000$. The frequency accuracy of the Cs atomic clock used in the satellite is generally $10^{-12}$; the equivalent frequency accuracy is one hundredth of a Hertz. Therefore, we use $0.006 \mathrm{~Hz}$ sinusoidal drift in the simulation to simulate if the satellite clock drift is appropriate. The curve of the satellite clock frequency drift set in the simulation is as shown in the left panel of Fig. 7.

(2) The curve of the user clock frequency drift $\Delta f_{r}(t)$

We set the frequency curve of the user clock as shown in the following formula:

$$
\Delta f_{r}(t)=5 \times \sin \left(2 \pi \frac{t}{5000}\right)
$$

where $t=1,2 \ldots 5000$. In the simulation, we assume that the receiver uses a normal crystal oscillator. Usually, the $10-\mathrm{MHz}$ crystal oscillator used by the receiver has a frequency accuracy of $10^{-8} \sim 10^{-9}$, and the equivalent frequency accuracy is several to several tens of Hertz. Therefore, we use $5 \mathrm{~Hz}$ sinusoidal drift in the simulation to simulate if the receiver crystal oscillator drift is appropriate. The curve of the user clock frequency drift set in the simulation is as shown in the middle panel of Fig. 7 .

(3) The curve of carrier frequency ionospheric influence $\Delta f_{\text {TEC }}(t)$

In the paper simulation, when the ionospheric influence is known, we set it as a constant. When the ionospheric effect is unknown with the solution, we use a slope-determined straight line to simulate which is as the following Formula (44). The curve of carrier frequency ionospheric influence in the simulation is as shown in the right panel of Fig. 7 ,

$$
\Delta f_{\mathrm{TEC}}(t)=2 t / 5000
$$

In the simulation, we compare the set clock frequency drift of the satellite clock with the frequency drift of the satellite clock calculated by the method which is present in this paper, and the set drift of the receiver clock frequency and the receiver frequency drift calculated by the method which is present in this paper, then to verify the correctness and performance of the article algorithm. We divide the results into two parts. The first is the frequency transfer accuracy that the paper algorithm can achieve under ideal conditions without observation noise (because the observation equation is properly simplified and optimized in the paper). The second part is the accuracy of the frequency transfer that the paper algorithm can achieve when there is observed noise in the carrier observations. The setting of observation noise in this paper's simulation is based on the carrier loop noise of a general satellite navigation signal and is set to Gaussian noise with a mean value of 0 degrees and a variance of 15 degrees.

\subsubsection{Frequency transfer accuracy under ideal conditions without observation noise}

As shown below, Fig. 8 shows the frequency transfer accuracy of the relevant method proposed in the paper without observation noise case. Figure $8 \mathrm{a}$ is the satellite frequency drift calculation error under the conditions as shown in Section 3.1 of the paper. In this case, the ionospheric effects on the signal propagation path are known in advance, and Doppler and satellite clock drift are calculated at the same time. Figure $8 \mathrm{~b}$ is the satellite frequency drift calculation error under the conditions as shown in Section 3.2 of the paper. In this case, the ionospheric effects on the signal propagation path are unknown, and the ionospheric Doppler, relative motion Doppler, and satellite clock drift are calculated at the same time. Figure $8 \mathrm{c}, \mathrm{d}$ is the satellite frequency drift calculation error and user crystal frequency drift calculation error under the conditions as shown in Section 4 of the paper. In this case, Doppler which is between user and satellite, satellite clock drift, and user crystal frequency drift are calculated at the same time.

\subsubsection{Frequency transfer accuracy under real conditions with observation noise}

Figure 9 shows the frequency transfer accuracy under real conditions with observation noise; the order of the figures is the same as in Fig. 8. In the real conditions, the signal carrier observations are due to noise in the environment and contain observation noise. In this paper, based on the carrier loop noise of a general satellite navigation signal, we set the carrier frequency observation noise independent and with a mean value of 0 degrees and a variance of 15 degrees. 

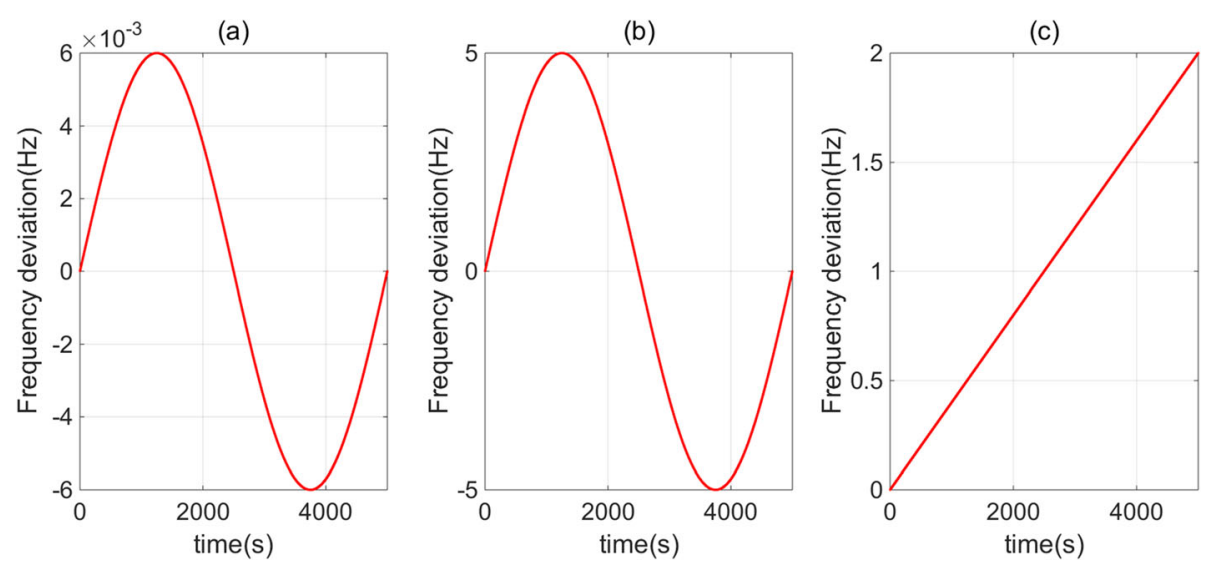

Fig. 7 The curve set in the simulation

From the above, simulation results can be seen as follows:

(1) From Fig. 8a, b, we can see that under ideal conditions without observation noise, the frequency transfer accuracy of the satellite can reach the order of $10^{-9} \mathrm{~Hz}$ in the case that "Frequency

Transmission Between MCS and Satellite combined RDSS and RNSS Signals."

(2) From Fig. 8c, d, we can see that under ideal conditions with observation noise, the frequency transfer accuracy of satellite and user crystal can reach the order of $10^{-7} \mathrm{~Hz}$ in the case that "Frequency Transmission Between MCS and user combined RDSS and RNSS Signals."

(3) From Fig. 9a, b, we can see that under real conditions with observation noise, the frequency transfer accuracy of the satellite can reach the order of $10^{-4} \mathrm{~Hz}$ in the case that "Frequency

Transmission Between MCS and Satellite combined RDSS and RNSS Signals."

(4) From Fig. 9c, d,we can see that under real conditions with observation noise, the frequency transfer accuracy of satellite and user crystal oscillator can reach the order of $10^{-3} \mathrm{~Hz}$ in the case that "Frequency Transmission Between MCS and user combined RDSS and RNSS Signals."

\subsubsection{The relationship between frequency and timing accuracy}

From Fig. 9, we can see that the frequency transfer error can reach $10^{-3} \sim 10^{-4} \mathrm{~Hz}$ in real condition, it is equivalent to $10 \mathrm{MHz}$ frequency accuracy to $10^{-13} \sim 10^{-14}$ which frequency accuracy is better than
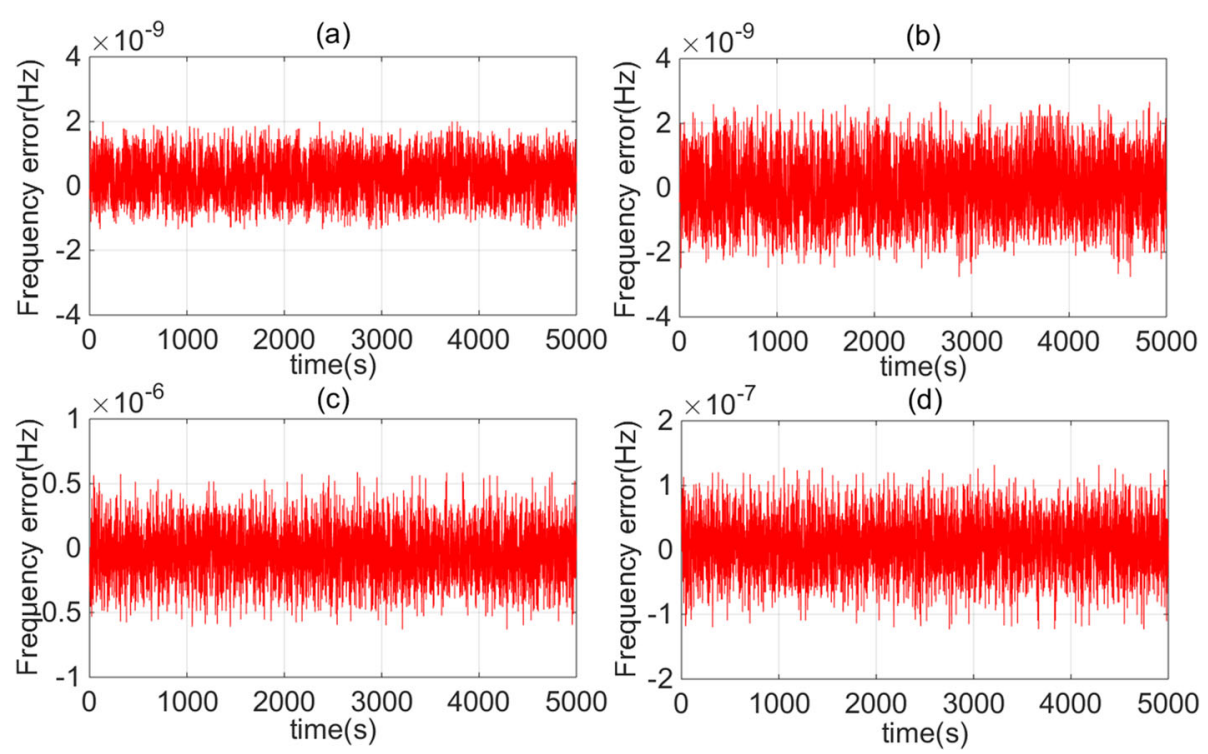

Fig. 8 Frequency transfer error curve under ideal conditions without observation noise 

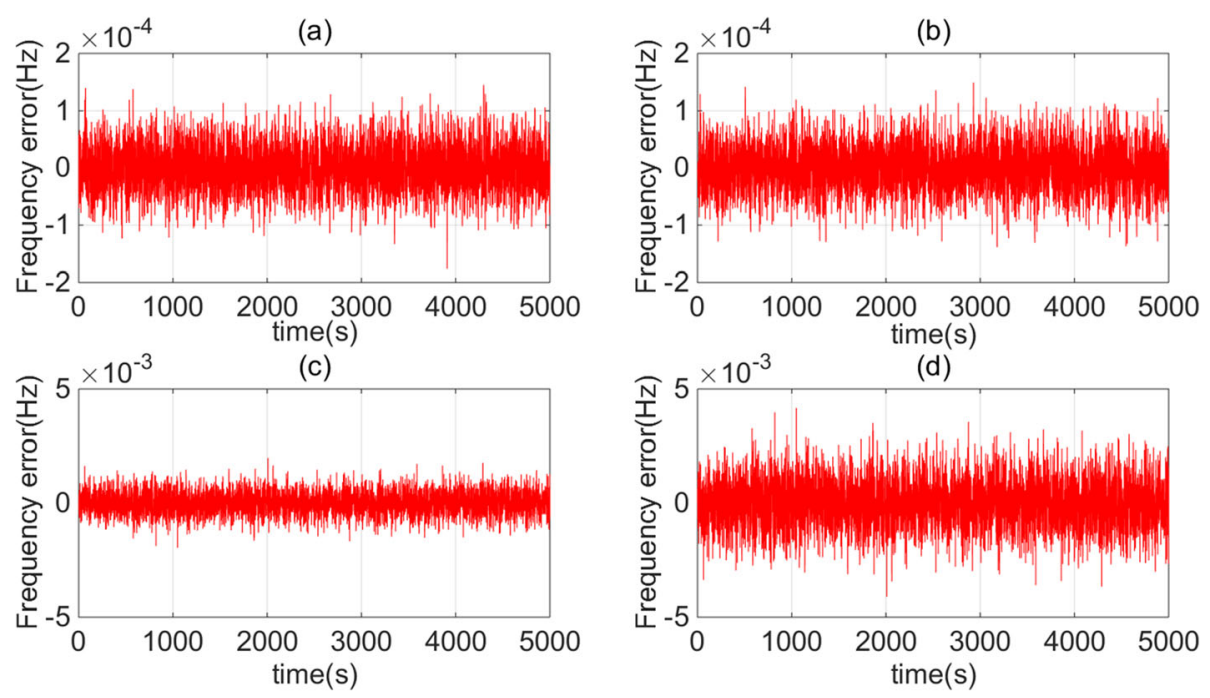

Fig. 9 Frequency transfer error curve under real conditions with observation noise

that of the Cs clock, and its frequency does not have long-term drift over time which is equivalent to a local accurate and stable frequency signal. When we use this frequency signal combined with pseudorange observation of RNSS signals shown in Figs. 4 and 6, we can effectively reduce the observation noise of RNSS signals, thereby improving timing accuracy. According to the results of the relevant paper [15-18], the time synchronization accuracy can reach several ns level.

\section{Conclusions}

The theoretical analysis and computer simulation result in this paper show that the combination of BeiDou's RDSS and RNSS can realize precision frequency transmission of BeiDou's MCS and satellites. The frequency transmission accuracy can reach $10^{-4} \mathrm{~Hz}$ under typical noisy conditions. Based on the combination of BeiDou's RDSS and RNSS signals, it also can realize precision frequency transfer between BeiDou's MCS and users, and its frequency transmission accuracy can reach $10^{-4} \sim 10^{-3} \mathrm{~Hz}$ under noisy conditions. However, the frequency accuracy of Cs atomic clock satellite generally can reach $10^{-12}$, equivalent to $10^{-2}$ $\mathrm{Hz}$ under the $10 \mathrm{MHz}$ oscillator, and the accuracy of the receiver crystal oscillator is typically $10^{-9} \sim 10^{-8}$, equivalent to $10 \mathrm{~Hz}$ under the $10 \mathrm{MHz}$ crystal oscillator. As a result, a more accurate frequency reference was provided for the satellite and receiver. Further, we combine RNSS pseudorange information to achieve ns order ultra-accuracy time synchronization of satellites and MCS, MCS, and users.

By further analyzing the frequency residual of the user receiver crystal oscillator, it can be seen that for the user receiver, using RDSS and RNSS signal of
BeiDou's GEO satellite can achieve the receiver crystal oscillator frequency drift calculation. The solution residual of the receiver crystal oscillator frequency can be seen as zero-mean and independent distributed for each BeiDou's GEO satellite, because for every GEO satellite, the RDSS and RNSS signal links of each satellite are independent of each other. Therefore, referring to the GPS AV processing algorithm $[19,20]$, if multiple satellites' RDSS and RNSS signals are used, a more accurate clock drift can be obtained through synthesis processing deviations of each GEO satellite calculated drift to further improve the frequency accuracy.

Different from GPS, GLONASS, and Galileo system, the BeiDou system not only broadcasts RNSS navigation signals, but also broadcasts RDSS signals on GEO satellites, and RDSS and RNSS signals are common to the clock source on the satellite. The precise frequency transfer method which is proposed in the paper uses BeiDou RDSS and RNSS signals and can realize the precision frequency transmission of BeiDou MCS and satellites, also BeiDou MCS and user, even when the receiver only uses cheap TCXO. The paper provides a feature of the joint application of the RDSS and RNSS signals of the BeiDou satellite navigation system, which is not available in other satellite navigation systems such as GPS.

\section{Abbreviations}

AV: ALL in View; BDS: BeiDou system; CV: Common view; GEO: Geostationary earth orbit; GNSS: Global Navigation Satellite System; GPS: Global Positioning System; IGSO: Inclined geosynchronous orbit; MCS: Master Control Station; MEO: Medium earth orbit; RDSS: Radio Determination Satellite Service; RNSS: Radio Navigation Satellite Service 


\section{Acknowledgements}

The author wants to thank the author's organization because they have provided us with many conveniences.

\section{About the authors}

Wenxue Liu was born in Weinan, Shaanxi, People's Republic of China, in 1985. He is a Ph.D. student. He has obtained his Bachelor's Degree in the University of Chinese Academy of Sciences, People's Republic of China in 2010. His current research focuses mainly involve GNSS application in time synchronization, etc.

Hong Yuan was born in Xi'an, Shaanxi, People's Republic of China, in 1968. $\mathrm{He}$ is a researcher and doctoral tutor of the Institute of Optoelectronics of the Chinese Academy of Sciences. He has been engaged in ionospheric wave propagation, GPS, BeiDou satellite navigation system construction, manned space application system projects, ionospheric physics, and ionospheric detection.

Jian Ge was born in Beijing, People's Republic of China, in 1971. He is a senior engineer and doctor of the Institute of Optoelectronics of the Chinese Academy of Sciences. His main research interests are satellite navigation receivers, satellite navigation signal systems, and high-precision time and frequency synchronization.

Ying Xu was born in Chengdu, Sichuan, People's Republic of China, in 1983. She is a researcher and doctor of the Institute of Optoelectronics of the Chinese Academy of Sciences, whose main research direction is satellite navigation and positioning technology, navigation signal system, and navigation enhancement technology.

\section{Authors' contributions}

HY proposed the conception of the possibility and concept of the joint application of BDSS and RNSS in BeiDou system. WX L contributed to the analysis and simulation. JG put forward relevant refinement suggestions for the computer simulation verification part of the article. It is recommended to define it as the simulation verification of the algorithm and YX puts forward constructive comments on the theoretical analysis part of the article. All authors read and approved the final manuscript.

\section{Funding}

This research was supported by the Beijing Municipal Science \& Technology Commission 5G special project (Z181100003218009), the National High Technology Research and Development Program ("863"-Program) of China (NO. 2015AA124101), and the Institute Innovation Project of Institute of Optics, Chinese Academy of Sciences "Research on Joint Tracking Technology of GNSS Dual Frequency Signals" (NO. Y70B12A1BY).

\section{Availability of data and materials}

We can provide the data.

Ethics approval and consent to participate

Approved.

\section{Consent for publication}

Approved.

\section{Competing interests}

The authors declare that they have no competing interests.

\section{Author details}

'Academy of Opto-Electronics, Chinese Academy of Sciences, No. 9, Deng Zhuang South Road, HaiDian District, Beijing 100094, People's Republic of China. ${ }^{2}$ School of Electronic, Electrical and Communication Engineering (EECE), University of Chinese Academy of Sciences, No. 19, Yuquan Road, Shijingshan District, Beijing 100049, People's Republic of China.

Received: 7 February 2019 Accepted: 10 September 2019

\section{Published online: 04 November 2019}

\section{References}

1. China Satellite Navigation Office, Report on the Development of BeiDou Navigation Satellite System (Version 2.1) (2012)
2. F. Xie, J. Liu, R. Li, et al., A simultaneous multiple BeiDou signal acquisition algorithm for a software-based GNSS receiver. Optik 127(4), 1607-1614 (2016)

3. D.W. Allan, M.A. Weiss, in 34th Annual Symposium on Frequency Control. Accurate time and frequency transfer during common-view of a GPS satellite (1980), pp. 334-346

4. J. Ray, F. Arias, G. Petit, T. Springer, T. Schildknecht, J. Clarke, Johansson, Progress in carrier phase time transfer. GPS Solutions 4(4), 47-54 (2001)

5. Y. Ge, W.J. Qin, K. Su, et al., A new approach to real-time precise point positioning (PPP) timing with IGS real-time service (RTS) products. Meas. Sci. Technol. (2019)

6. Z. Jihai, G. Wei, W. Wenjun, et al., BDS PPP time transfer at NTSC [C]//2018 IEEE International Frequency Control Symposium (IFCS). IEEE, 1-6 (2018)

7. P. Zhang, R. Tu, Y. Gao, et al., Study of time link calibration based on GPS carrier phase observation. IET Radar, Sonar \& Navig 12(11), 1330-1335 (2018)

8. H. Yuan, W. Guang, in 2012 IEEE International Frequency Control Symposium Proceedings, IEEE. Common view time transfer by a GEO navigation satellite (2012), pp. 1-4

9. C.J. Dou, B.Q. Zhang, S.S. Tan, T. Long, in Education Technology and Computer Science (ETCS). A new three-satellite high-precision RDSS/RNSS combination positioning method, vol 2 (Second international workshop on 2010, March, 2010), pp. 288-290

10. L. Liu, T. Zhang, S. Zhou, et al., Improved design of control segment in BDS-3. Navigation 66(1), 37-47 (2019)

11. J.W. Betz, Engineering Satellite-Based Navigation and Timing: Global Navigation Satellite Systems, Signals, and Receivers (Wiley, 2016)

12. C.J. Dou, B.Q. Zhang, S.S. Tan, T. Long, in Computational and Information Sciences (ICCIS). The global search and rescue system of combined RDSS/ RNSS analysis (Fourth International Conference on 2012, August, 2012), pp. 795-798

13. P.M. Kintner, B.M. Ledvina, E.R. De Paula, GPS and ionospheric scintillations. Space Weather 5(9) (2007)

14. J.J. Spilker Jr., P. Axelrad, B.W. Parkinson, P. Enge, Global Positioning System: Theory and Applications, Volume I (1996), pp. 520-524

15. T. Krawinkel, S. Schön, Benefits of receiver clock modeling in code-based GNSS navigation. GPS Solutions 20(4), 687-701 (2016)

16. E. Byrne, T.Q. Nguyen, L. Boehnke, F. van Graas, S. Stein, Clock Steering Using Frequency Estimates from Stand-Alone GPS Receiver Carrier Phase Observations (Proceedings of the 44th Annual Precise Time and Time Interval Systems and Applications Meeting, Reston, 2012), pp. 211-220

17. B.M. Penrod, in Frequency Control Symposium, 1996. Adaptive temperature compensation of GPS disciplined quartz and rubidium oscillators (50th., Proceedings of the 1996 IEEE International. IEEE, 1996), pp. 980-987

18. M.A. Lombardi, A.N. Novick, V.S. Zhang, Characterizing the Performance of Gps Disciplined Oscillators with Respect to Utc (Nist) (2005), pp. 677-684

19. Z. Jiang, G. Petit, Time transfer with GPS satellites all in view. Proc. ATF 2004, 236 (2004)

20. D. Luna, D. Pérez, A. Cifuentes, et al., Three-cornered hat method via GPS common-view comparisons. IEEE Trans. Instrum. Meas. 66(8), 2143-2147 (2017)

\section{Publisher's Note}

Springer Nature remains neutral with regard to jurisdictional claims in published maps and institutional affiliations. 